\title{
Comportamento sexual de risco em jovens: aspectos cognitivos e emocionais
}

\author{
Ítala Raymundo Chinaz:o - Universidade Luterana do Brasil, Canoas, Rio Grande do Sul, Brasil \\ Sheila Goncalves Câmara - Universidade Luterana do Brasil, Canoas, Rio Grande do Sul, Brasil \\ Deise Gabriela Frantz - Universidade Luterana do Brasil, Canoas, Rio Grande do Sul, Brasil
}

\begin{abstract}
Resumo
O estudo avalia a contribuição dos construtos da Teoria do Comportamento Planejado e variáveis emocionais sobre a intenção de repetir o comportamento de manter relações sexuais sem preservativo entre jovens. Participaram 1.245 estudantes com idades entre 15 e 24 anos, de Canoas/RS. A prevalência do comportamento foi de 40\% (500 jovens), os quais constituíram a amostra. Os instrumentos usados foram: questionário baseado na Teoria do Comportamento Planejado aplicada ao comportamento e sobre a intensidade de emoções básicas antes, durante e depois da realização. Os dados foram analisados através de regressão linear múltipla. No modelo final, o construto atitude se apresentou como o maior preditor da intenção (80\%). As emoções de medo depois, alegria antes e culpa depois explicaram, conjuntamente, $12,5 \%$. Os resultados indicam a influência dos aspectos emocionais na realização do comportamento e revelam a prioridade do componente atitudinal na predição da intenção de realizar relações sexuais sem preservativo entre jovens.

Palavras-chave: Comportamento sexual, Assunção de riscos, Adolescência, Aspectos cognitivos, Emoções.
\end{abstract}

\section{Abstract}

Sexual risk behavior in youngsters: cognitive and emotional aspects

The study assesses the contribution of the Theory of Planned Behavior's constructs and emotional variables on the intention to repeat the behavior of maintain sexual intercourse without using condom among youngsters. Participated 1,245 students aged between 15 and 24 years, from Canoas/RS. The prevalence of the behavior was 40\% (500 youngsters), which constituted the sample. The instruments were: questionnaire on the Theory of Planned Behavior applied to behavior and on the intensity of basic emotions before, during and after implementation. Data were analyzed using multiple linear regression. In the final model, the attitude presented itself as the greatest predictor of intention (80\%). The emotions of fear after, joy before and guilt after, jointly, explained $12,5 \%$. Results indicate the importance of emotional aspects in the behavior realization, but reveal the priority of the attitudinal component in the prediction of the intention to have sex without condom among youngsters.

Keywords: Sexual behavior, Risk-taking, Adolescence, Cognitive aspects, Emotions.

\section{Resumen}

Conducta sexual de riesgo en jóvenes: aspectos cognitivos y emocionales

El estudio evalúa la contribución de los constructos de la Teoría del Comportamiento Planificado y variables emocionales sobre la intención de repetir la conducta de mantener relaciones sexuales sin preservativo entre jóvenes. Participaron 1245 estudiantes con edad entre 15 y 24 años, de Canoas/RS. La prevalencia de la conducta fue de 40\% (500 jóvenes), los cuales constituyeron la muestra. Los instrumentos fueron: cuestionario sobre la Teoría del Comportamiento Planificado aplicada a la conducta y sobre la intensidad de emociones básicas antes, durante y después de la realización. Los datos fueron analizados a través de regresión lineal múltiple. En el modelo final la actitud presentóse como el mayor predictor de la intención (80\%). Las emociones de miedo después, alegría antes y culpa después explicaron conjuntamente el 12,5\%. Los resultados indican la importancia de los aspectos emocionales en la realización de la conducta, pero revelan la prioridad del componente actitudinal en la predicción de la intención de mantener relaciones sexuales sin preservativo entre jóvenes.

Palabras-clave: Conducta sexual, Assunción de riesgos; Adolescencia, Aspectos cognitivos, Emociones.

\section{Introdução}

O conceito de comportamento de risco diz respeito à participação em atividades que possam comprometer a saúde física e mental do indivíduo (Feijó \& Oliveira, 2001). Dentre os comportamentos de risco verificados na juventude, a conduta sexual desprotegida é bastante prevalente entre os indivíduos sexualmente ativos (Camargo \& Botelho, 2007; Campo-Arias, Ceballo, \& Herazo, 2010). O comportamento sexual é considerado arriscado quando os indivíduos não utilizam o preservativo para evitar gestação indesejada e/ou proteger-se da contaminação por doenças sexualmente transmissíveis (DSTs), por exemplo, a Síndrome da Imunodeficiência Adquirida (HIV/AIDS) (Cruzeiro e colaboradores, 2010).

No Brasil, a incidência de casos de HIV/AIDS notificados até metade do ano de 2011, entre jovens de 15 e 24 anos, equivalia a 1.401. Destes, 175 casos $(12,49 \%)$ eram do Rio Grande do Sul, caracterizando o estado como o segundo com maior incidência da doença entre os jovens. Percebe-se que a via prevalente de transmissão, nessa faixa etária, se dá pela relação sexual tanto em homens (52,3\%) quanto em mulheres (75,9\%) (Brasil, 2011).

No que se refere à gravidez na adolescência, em todo o mundo, $11 \%$ dos partos anuais são de adolescentes com idades entre 15 e 19 anos. Metade desses nascimentos 
acontece nos países Bangladesh, Brasil, República Democrática do Congo, Etiópia, Índia, Nigéria e Estados Unidos (World Health Organization [WHO], 2011). Dados do Ministério da Saúde do Brasil, referentes ao segundo semestre de 2009, demonstraram que houve uma redução no número de meninas grávidas, na faixa etária de 10 a 19 anos, nos últimos dez anos. Entre 1998 e 2008 , houve uma redução de 30,6\% no número de partos (Brasil, 2009). No entanto, o número de quase meio milhão de adolescentes que engravidaram em 2008 é ainda grandioso, trazendo questionamentos sobre suas razões, riscos e consequências (Neto, Dias, Rocha, \& Cunha 2007).

Estudos com adolescentes mostram que grande parte dos jovens sexualmente ativos faz uso do preservativo ocasionalmente (Andrade e colaboradores, 2009; Cruzeiro e colaboradores, 2010; Ferreira \& Torgal, 2011). Em pesquisa realizada por Orlandi e Toneli (2008) com adolescentes pais de Santa Catarina, foi identificado uso descontínuo de preservativo e certa despreocupação com contaminações por HIV/AIDS e outras DSTs, tendo como justificativa a confiança na fidelidade do parceiro. Estudo realizado por Teixeira, Knauth, Fachel e Leal (2006) com adolescentes de três capitais brasileiras demonstrou que, na primeira relação sexual da vida, considerando-se apenas os que haviam utilizado algum tipo de método, o preservativo apareceu em primeiro lugar e, em segundo, o anticoncepcional oral. Já na relação sexual mais recente, o uso de preservativo diminuiu, tendo o anticoncepcional oral se tornado o método mais usual. Há, portanto, uma menor preocupação com a contaminação por DST-HIV/AIDS no decorrer do tempo, enquanto permanece e/ou aumenta a preocupação com a gravidez indesejada. As autoras consideram que o uso do preservativo está vinculado a fatores socioculturais, mas, também, de ordem situacional e individual. Em estudo com adolescentes holandeses, Empelen e Kok (2008) também identificaram que o fato de contar com parceiros estáveis diminuía o uso de preservativo.

Segundo Sampaio Filho e colaboradores (2010), no período da adolescência, ainda existe certa imaturidade quanto ao pensamento abstrato, o que pode fazer com que o adolescente não considere sua vulnerabilidade, expondo-se a riscos sem avaliar ou prever as consequências. Assim, os jovens podem ter uma percepção distorcida do risco real da infecção por DST-HIV/AIDS nas relações sexuais, pensando que este é um perigo impossível ou altamente improvável. Nesse sentido, Oliveira, Dias e Silva (2005) encontraram, no contexto de adolescentes da Paraîba/BR, que a atitude em relação ao comportamento de não uso de preservativo estava associada à intenção de não uso posteriormente à primeira experiência. No caso do estudo de Jemmont III e colaboradores (2007), na África do Sul, a pressão social e a norma subjetiva demonstraram-se especialmente relevantes. Quanto ao tempo de experiência no comportamento, Empelen e Kok (2008) identificaram que comportamentos anteriores com consequências positivas contribuíam para aumentar a intenção de realização do comportamento novamente.

Para a avaliação de comportamentos de saúde, como é o caso do uso de preservativo (Serovich \& Greene, 1997), a Teoria do Comportamento Planejado (TCP) tem sido amplamente utilizada (Armitage, Conner, Loach, \& Willets, 1999; Bennet \& Bozionelos, 2000; Matos, Veiga, \& Reis, 2009; Mendes, Rodrigues, Cornélio, Gallani, \& Godin, 2010). A TCP foi desenvolvida com o objetivo de explicar o comportamento social, sendo, posteriormente aplicada ao âmbito da saúde, com o objetivo de compreender por que as pessoas executam comportamentos de saúde. A teoria considera que a maior parte dos comportamentos sociais importantes está submetida à vontade da pessoa. Nesse sentido, a intenção de realização corresponde ao determinante mais imediato de um comportamento (León Rubio \& Medina Anzano, 2002).

A intenção é precedida por atitudes, norma subjetiva e controle percebido. As atitudes correspondem a um fator pessoal e são conceitualizadas como a avaliação realizada para concluir, se realizar uma ação determinada implicará uma consequência positiva ou negativa. A norma subjetiva define-se como a pressão social ou influência da percepção subjetiva dos outros sobre a realização do comportamento. A atitude e a norma subjetiva dependem, portanto, da percepção das consequências resultantes de realizar uma conduta e da percepção do que se acredite que pensam os outros sobre essa conduta (Ajzen \& Fishbein, 1980; Terry, Gallois, \& McCamish, 1994). O controle percebido equivale ao grau de facilidade/dificuldade percebido pelo sujeito em executar ou não o comportamento (Ajzen, 2002; Matos e colaboradores, 2009). Está relacionado ao conceito de autoeficácia percebida (Bandura, 2000), sendo que, além da intenção de realizar um comportamento, é preciso que a pessoa se sinta capaz de executá-lo (Figura 1).

A TCP considera que diversos fatores, como os objetivos da pessoa, personalidade, aspectos sociodemográficos, entre outros, relacionam-se aos 


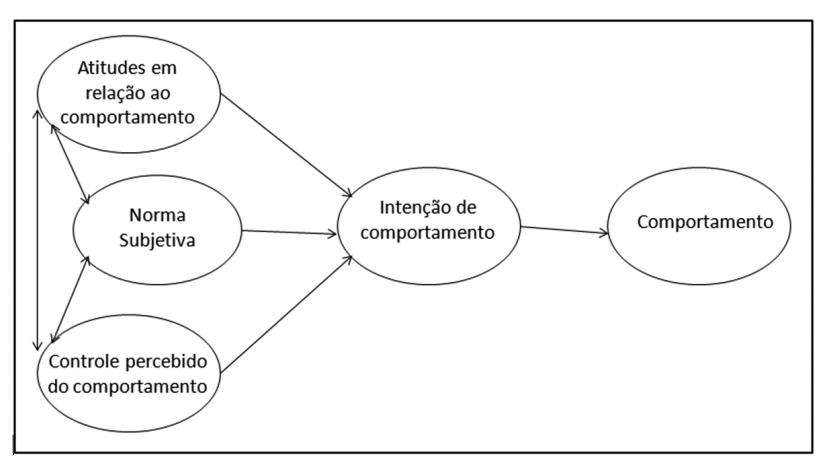

Figura 1. Esquema das principais características da Teoria do Comportamento Planejado. Adaptado de León Rúbio e Medina Alzano (2002, p. 90)

comportamentos de saúde; no entanto, tais variáveis são externas ao modelo. O modelo da TCP consiste em um processamento cognitivo acerca da execução de um comportamento em um contexto social. Dessa forma, contempla fatores intervenientes externos, sem desconsiderar a interferência de aspectos internos (León Rubio \& Medina Anzano, 2002).

No campo dos comportamentos de risco, a utilização da TCP tem se dado especialmente em relação ao consumo de drogas (Armitage e colaboradores, 1999; Gouveia, Pimentel, Leite, Albuquerque, \& Costa, 2009) e às relações sexuais/uso de preservativo (Bennet \& Bozionelos, 2000; Matos e colaboradores, 2009). O uso de preservativos é um comportamento complexo que envolve outros fatores que não somente o conhecimento teórico acerca da importância da proteção do preservativo. Dados epidemiológicos do Ministério da Saúde demonstram que, embora os jovens tenham mais conhecimento sobre os benefícios do uso de preservativos, muitos ainda não utilizam (Brasil, 2010). Em estudo longitudinal comparando os sexos, Broaddus, Schmiege e Bryan (2011) verificaram incoerências entre a intenção e a realização do comportamento de não uso de preservativo em ambos os sexos.

Com respeito a outros possíveis fatores envolvidos na tomada de decisão sobre a execução do ato em si e na sua manutenção ou extinção, os aspectos emocionais representam um fator importante a ser considerado (Ballester \& Asensio, 2001). As emoções desviam o curso do comportamento, conforme um planejamento racional, podendo dar uma nova orientação ao mesmo (Mérida \& Jorge, 2010). Conforme Caffray e Schneider (2000), os adolescentes são motivados a executar determinados comportamentos, mesmo que sejam de risco, para aumentar ou evitar determinadas emoções.
O envolvimento em comportamentos de risco está associado à busca de emoções positivas e à evitação de sentimentos negativos, sendo que tal excitação funciona como reforçador para executar novamente a conduta.

Este estudo procurou identificar aspectos cognitivos e emocionais associados à intenção de repetição do comportamento sexual de risco, de não uso de preservativo, na população de adolescentes estudantes que apresentaram tal comportamento no último ano. Para tanto, variáveis relativas à TCP e a emoções básicas antes, durante e depois da realização foram analisadas por etapas a fim de identificar sua capacidade preditiva sobre a intenção.

\section{Método}

\section{Participantes}

Neste estudo utilizou-se o delineamento transversal, com uma amostra de jovens estudantes do terceiro ano do ensino médio regular em turno diurno da rede pública estadual do município de Canoas/RS, com idade entre 16 e 24 anos. A população de alunos matriculados nesse nível de ensino correspondeu a 2.338 estudantes das 17 escolas da rede (ensino regular no turno diurno). Para o cálculo de amostra, utilizou-se o programa Epi-Info, considerando-se o total de escolares matriculados e uma prevalência esperada para a variável dependente (intenção de repetição do comportamento sexual de risco) de $50 \%$, um erro máximo tolerado de $\pm 3 \%$ e um nível de confiança de $95 \%$. Com isso, a amostra inicialmente calculada foi de 733 estudantes. Considerando-se um possível efeito de delineamento de 1,5 , essa amostra foi ampliada em 367 sujeitos, acrescidos de mais $20 \%$ (147 sujeitos) para suportar as perdas. Com isso, o tamanho da amostra foi estimado em 1.247 estudantes. Como esses representavam cerca da metade da população-alvo, realizou-se o sorteio da metade mais uma das turmas existentes em cada escola, obtendo-se 1.277 alunos. Após a exclusão de 32 sujeitos por idade superior a 24 anos ou por não preenchimento adequado do instrumento de pesquisa, obteve-se um total de 1.245 casos válidos. Desses jovens, 500 (40,2\%) mantiveram relações sexuais sem preservativo no último ano e constituíram a amostra do presente estudo.

Houve predomínio do sexo feminino (64\%), e a média de idade foi de 17,9 anos $(D P=1,60)$. A maioria $(63,6 \%)$ mora com os pais. Quanto à renda familiar, para os $62,2 \%$ dos jovens que responderam a esta questão, a média de rendimentos ficou em 3,23 ( $D P=2,11)$ salários-mínimos nacionais. Dentre os jovens, 47,6\% 
somente estudavam, enquanto $48,22 \%$ estudavam e trabalhavam e 4,2\% dos sujeitos não responderam.

\section{Instrumentos}

Questionário sociodemográfico. Desenvolvido para o presente estudo, reúne informações sobre sexo, idade, com quem o jovem vive, renda familiar e situação ocupacional.

Questionário embasado nos construtos da TCP, com questões de caráter recordatório sobre emoções básicas antes, durante e depois do comportamento sexual de risco realizado anteriormente. $O$ instrumento foi adaptado para estudantes de ensino médio brasileiros, a partir dos estudos de Sanchez, Caballero, Carrera, Blanco e Pizarro (2001) com estudantes madrilenhos. A versão brasileira foi avaliada por três psicólogos sociais brasileiros com conhecimento sobre a TCP e processos sociais básicos, como é o caso das emoções relacionadas ao contexto e à situação. Dada a simplicidade do instrumento (duas questões por construto da TCP e avaliação de intensidade das emoções básicas em três tempos), este foi considerado de fácil entendimento para a população-alvo. Antes da aplicação definitiva, o instrumento foi submetido à análise de estudantes de terceiro ano do ensino médio de uma escola da rede pública de Cachoeirinha/RS. Um grupo de 13 estudantes respondeu ao instrumento, apontando dúvidas e dificuldades encontradas por escrito (análise semântica). Em seguida, tais aspectos foram debatidos em grupo com os pesquisadores. Não houve problemas semânticos ou de entendimento das questões, no entanto, foram modificadas as questões referentes à norma subjetiva - família, a fim de que os jovens compreendessem que era uma avaliação pessoal deles sobre a percepção familiar e não o posicionamento concreto da família sobre o tema.

O instrumento foi, então, aplicado em 78 jovens estudantes de terceiro ano do ensino médio de escolas públicas estaduais de Cachoeirinha/RS (estudo piloto) diferentes da escola na qual foi realizada a análise semântica. As variáveis referentes aos construtos da TCP atitude, controle percebido, norma subjetiva e intenção de repetir o comportamento no futuro obtiveram um coeficiente alfa de Cronbach de 0,73. Já a intensidade recordada das emoções básicas antes, durante e depois da realização do comportamento obteve um índice de consistência interna de 0,79. De acordo com os procedimentos realizados para a avaliação das propriedades psicométricas do instrumento, a versão brasileira contou com seis dimensões, divididas em dois blocos (construtos da TCP e emoções básicas), discriminados a seguir.

\section{Construtos da TCP}

1) Experiência prévia: frequência com que já realizou o comportamento no último ano, relação com parceiro(s), utilização de outros métodos para prevenção de gravidez e tipo de método utilizado; 2) Atitude: percepção de consequências (variável de um - muito positivas, a sete - muito negativas); importância/relevância do comportamento (de um - nenhuma, a sete - muita). A atitude foi avaliada pela média entre as duas variáveis avaliadas; 3) Controle percebido: o quanto o comportamento é avaliado como controlável (variável de um - nada, a sete - totalmente); 4) Norma subjetiva: avaliação da percepção da família sobre o comportamento (variável de um - muito negativa, a sete - muito positiva); avaliação da percepção dos amigos sobre o comportamento (variável de um - muito negativa, a sete - muito positiva); acordo com a percepção da família (variável de um - acordo total, a sete - desacordo total); acordo com a percepção dos amigos (variável de um - acordo total, a sete - desacordo total). A norma subjetiva foi avaliada pela média entre as quatro variáveis avaliadas; 5) Intenção de repetir o comportamento no futuro (variável de um - nenhuma a sete - muita). A consistência interna dos construtos da TCP (dimensões 2 a 5) foi de 0,70 no presente estudo.

\section{Emoções básicas}

Intensidade recordada de cada uma das emoções básicas de alegria, tristeza, raiva, culpa e medo em três momentos - antes, durante e depois da realização do comportamento (variável de um - nenhuma, a sete muito intensa). A escala total, com 15 itens, alcançou, no presente estudo, um coeficiente alfa de Cronbach de 0,80 .

\section{Procedimentos}

As escolas foram contatadas para apresentação do projeto de pesquisa, ocasião em que foi obtida autorização para a sua realização. Combinou-se uma data para a coleta de dados e, no dia anterior, os pesquisadores entraram nas turmas selecionadas para convidar os alunos, explicar-lhes os objetivos da pesquisa, obter o assentimento dos mesmos e encaminhar o Termo de Consentimento Livre e Esclarecido (TCLE), a ser trazido no dia seguinte, assinado pelos responsáveis. Os dados foram coletados nas salas de aula, com tempo médio de 30 minutos, por alunos bolsistas de iniciação 
científica da ULBRA/Canoas, devidamente capacitados para a atividade. Houve até três retornos a cada escola, em semanas subsequentes, para coletar os dados entre os alunos ausentes e aqueles que não haviam trazido o TCLE assinado pelos responsáveis.

De acordo com os aspectos éticos e legais na pesquisa científica, foi desenvolvido o TCLE para que os jovens tomassem conhecimento, por escrito, dos objetivos da pesquisa e assinassem (no caso de maiores de 18 anos) ou trouxessem o documento assinado pelos responsáveis (no caso dos menores de idade), manifestando acordo em participar do estudo. O projeto foi aprovado pelo Comitê de Ética da instituição de afiliação dos autores (Protocolo 2004-107H).

Foi realizada análise descritiva para descrever a população em estudo e caracterizar o comportamento de manter relações sexuais sem preservativo entre os participantes. A Correlação de Pearson foi utilizada para avaliar a relação entre as variáveis da TCP (excluindo-se a variável dependente intenção), e emoções e intenção. Foram selecionadas para as análises de regressão somente aquelas cujo $r$ foi igual ou superior a 0,20 . A análise multivariada foi realizada por etapas, conforme é apresentado a seguir:

1) Análise de regressão linear múltipla $(\phi \leq 0,05)$ para avaliar a contribuição das variáveis da TCP para intenção de repetição do comportamento, com controle das variáveis sexo e idade; e, análise de regressão linear múltipla para avaliar a contribuição das variáveis relacionadas às emoções para intenção de repetição do comportamento, com controle das variáveis sexo e idade.

2) Análise de regressão linear múltipla $(\phi \leq 0,05)$ para avaliar a contribuição conjunta das variáveis da TCP e das relacionadas às emoções para intenção de repetição do comportamento, na configuração de um modelo final, também com controle das variáveis sexo e idade. Foram utilizadas, como variáveis independentes somente aquelas que contribuíram significativamente nos dois modelos antecedentes.

\section{Resultados}

Sobre a frequência de relações sexuais sem preservativos no último ano, 49,4\% dos jovens referiram ter mantido relações sexuais sem preservativo poucas vezes ou apenas em uma ocasião isolada; 7,5\%, a metade das vezes que manteve relações sexuais e 43,1\%, quase sempre ou sempre. Das relações desprotegidas,
$83 \%$ foram com parceiro(a) estável, 11,4\% com pessoa conhecida e 5,6\% com parceiro(a) esporádico. Como prevenção de gravidez, $75 \%$ dos jovens utilizaram algum método contraceptivo, sendo que o mais utilizado foi a pílula $(83,5 \%)$ e os métodos naturais, como coito interrompido (6,3\%). Como um método não contraceptivo, mas abortivo, a pílula do dia seguinte foi referida por 10,3\% dos jovens. Como a intenção de repetir o comportamento no futuro foi avaliada de forma contínua (variando de um - nenhuma a sete muita), a média de intenção manifestada pelos jovens foi de 3,86 ( $D P=2,25)$, revelando, no contexto da escala utilizada, uma intenção média de voltar a repetir o comportamento.

$\mathrm{Na}$ correlação de Pearson, entre os construtos da TCP emoções e intenção de repetição do comportamento sexual de risco, foram selecionadas as variáveis atitude, norma subjetiva, medo antes, medo durante, medo depois, tristeza depois, alegria antes, alegria durante, alegria depois, raiva depois, culpa antes, culpa durante e culpa depois como fatores em estudo para as análises de regressão subsequentes. Embora outras variáveis tenham se apresentado como significativas, considerou-se muito baixa a correlação, considerando o ponto de corte estabelecido de $r \geq 0,20$ (Tabela 1).

O modelo obtido por regressão múltipla, tendo como fatores em estudo somente as variáveis da TCP, apresentou capacidade explicativa de $35 \%$ sobre a intenção, tendo associação significativa com a intenção de repetir o comportamento de manter relações sexuais sem o uso de preservativo as variáveis: idade, atitude e norma subjetiva (Tabela 2).

No modelo resultante da análise de regressão múltipla, que teve como fatores em estudo somente as variáveis relacionadas às emoções, encontrou-se capacidade explicativa de $24 \%$ sobre a intenção e as variáveis com associação significativa foram: idade, culpa depois, alegria antes e medo depois.

O modelo final, resultante da análise conjunta das variáveis com associação significativa à intenção de repetição do comportamento no futuro, alcançou capacidade explicativa de $40 \%$ sobre a intenção de repetir o comportamento de manter relações sexuais sem o uso de preservativo (Tabela 4).

A atitude foi o preditor mais potente, seguido do medo depois da realização do comportamento, alegria antes, norma subjetiva e culpa depois. A idade correspondeu a $2 \%$ da explicação. O modelo obtido indica que atitudes positivas, menor medo depois, maior alegria antes, norma subjetiva favorável ao comportamento, 
Tabela 1. Correlação dos aspectos cognitivos/emocionais com intenção de manter relações sexuais desprotegidas $(\mathrm{n}=500)$

\begin{tabular}{|c|c|}
\hline Variáveis & Intenção \\
\hline \multicolumn{2}{|l|}{ Variáveis da TCP } \\
\hline Atitude & $-0,580^{* *}$ \\
\hline Norma subjetiva & $0,375^{* *}$ \\
\hline Controle percebido & $-0,136^{* *}$ \\
\hline \multicolumn{2}{|l|}{ Experiência emocional } \\
\hline Medo antes & $-0,264^{* *}$ \\
\hline Medo durante & $-0,244^{* *}$ \\
\hline Medo depois & $-0,393^{* *}$ \\
\hline Tristeza antes & $-0,154^{* *}$ \\
\hline Tristeza durante & $-0,156^{* *}$ \\
\hline Tristeza depois & $-0,263^{* *}$ \\
\hline Alegria antes & $0,228^{* *}$ \\
\hline Alegria durante & $0,209^{* *}$ \\
\hline Alegria depois & $0,328^{* *}$ \\
\hline Raiva antes & $-0,081$ \\
\hline Raiva durante & $-0,104^{*}$ \\
\hline Raiva depois & $-0,213^{* *}$ \\
\hline Culpa antes & $-0,263^{* *}$ \\
\hline Culpa durante & $-0,284^{* *}$ \\
\hline Culpa depois & $-0,402^{* *}$ \\
\hline
\end{tabular}

$*_{\mathrm{p}} \leq 0,05 ; * *_{\mathrm{p}} \leq 0,01$

Tabela 2. Aspectos cognitivos associados à intenção de manter relações sexuais desprotegidas, controlados por sexo e idade $(\mathrm{n}=500)$

\begin{tabular}{lcccccc}
\hline Variáveis (TCP) & \multicolumn{5}{c}{ Intenção de voltar a manter relações sexuais sem preservativo } \\
\cline { 2 - 7 } & $\mathrm{R}$ & $\mathrm{R} 2$ & Beta & $\mathrm{t}$ & \multicolumn{1}{c}{$\boldsymbol{p}$} & $\mathrm{IC}(95 \%)$ \\
\hline Sexo & 0,03 & 0,00 & 0,02 & 0,67 & 0,50 & $(-0,22-0,46)$ \\
Idade & 0,13 & 0,01 & 0,07 & 2,07 & 0,04 & $(0,01-0,21)$ \\
Atitude & 0,57 & 0,33 & $-0,51$ & $-12,70$ & 0,00 & $(-0,81--0,59)$ \\
Norma subjetiva & 0,59 & 0,35 & 0,13 & 3,22 & 0,00 & $(0,10-0,41)$ \\
\hline
\end{tabular}

Tabela 3. Aspectos emocionais associados à intenção de manter relações sexuais desprotegidas, controlados por sexo e idade $(n=500)$

\begin{tabular}{lcccccc}
\hline Variáveis (emoções) & \multicolumn{5}{c}{ Intenção de voltar a manter relações sexuais sem preservativo } \\
\cline { 2 - 7 } & $\mathrm{R}$ & $\mathrm{R} 2$ & Beta & $\mathrm{t}$ & \multicolumn{1}{c}{$\mathrm{I}(95 \%)$} \\
\hline Sexo & 0,03 & 0,00 & 0,04 & 1,03 & 0,30 & $(-0,18-0,58)$ \\
Idade & 0,13 & 0,01 & 0,10 & 2,47 & 0,01 & $(0,03-0,26)$ \\
Culpa depois & 0,41 & 0,16 & $-0,24$ & $-4,57$ & 0,00 & $(-0,31--0,12)$ \\
Alegria antes & 0,46 & 0,21 & 0,21 & 5,06 & 0,00 & $(0,13-0,29)$ \\
Medo depois & 0,49 & 0,24 & $-0,21$ & $-4,08$ & 0,00 & $(-0,29--0,10)$ \\
\hline
\end{tabular}


Tabela 4. Aspectos cognitivos/emocionais associados à intenção de manter relações sexuais desprotegidas, controlados por sexo e idade $(n=500)$

\begin{tabular}{lcccccc}
\hline \multirow{2}{*}{$\begin{array}{l}\text { Fatores em estudo (TCP } \\
\text { e emoções) }\end{array}$} & $\mathrm{R}$ & $\mathrm{R} 2$ & Beta & $\mathrm{t}$ & \multicolumn{1}{c}{ Intenção de } & \multicolumn{2}{c}{$\mathrm{IC}(95 \%)$} \\
\cline { 2 - 6 } & 0,04 & 0,00 & 0,02 & 0,65 & 0,51 & $(-0,23-0,46)$ \\
Sexo & 0,14 & 0,02 & 0,09 & 2,36 & 0,01 & $(0,0-0,22)$ \\
Idade & 0,58 & 0,34 & $-0,42$ & $-9,45$ & 0,00 & $(-0,6--0,45)$ \\
Atitude & 0,61 & 0,37 & $-0,10$ & $-2,04$ & 0,04 & $(-0,1-0,00)$ \\
Medo depois & 0,62 & 0,38 & 0,12 & 3,28 & 0,00 & $(0,0-0,20)$ \\
Alegria antes & 0,62 & 0,39 & 0,10 & 2,33 & 0,02 & $(0,0-0,35)$ \\
Norma subjetiva & 0,63 & 0,40 & $-0,10$ & $-2,13$ & 0,03 & $(-0,1--0,01)$ \\
Culpa depois & & & & & & \\
\hline
\end{tabular}

menor culpa depois e maior idade contribuem para uma maior intenção de manter novamente relações sexuais sem preservativo na população em estudo.

\section{Discussão}

O presente estudo visou avaliar, conjuntamente, aspectos cognitivos e emocionais relacionados à prática do comportamento de não uso de preservativo em relações sexuais entre jovens escolares. No que tange ao comportamento sexual, a atividade propriamente dita corresponde a um aspecto relativamente novo na vida dos jovens. Diversos processos ocorrem simultaneamente: a sexualidade madura, as relações afetivas e, no caso das relações sexuais, o uso de preservativo (Matos e colaboradores, 2009). Este traz consigo a responsabilização e o receio vinculado à própria saúde e à procriação.

O uso de preservativo, portanto, implica em diversos fatores que ultrapassam o componente informacional. Dados epidemiológicos do Ministério da Saúde do ano de 2010 (Brasil, 2010) demonstram que os jovens têm, atualmente, mais conhecimento sobre a prevenção da HIV/AIDS e outras DSTs. Entretanto, não se percebe um comportamento preventivo consistente nessa faixa etária (Campo-Arias e colaboradores, 2010; Cruzeiro e colaboradores, 2010; Ferreira \& Torgal, 2011).

O presente estudo revelou um modelo explicativo composto por duas variáveis cognitivas, as atitudes e a norma subjetiva, conjuntamente com as variáveis emocionais de medo depois, alegria antes e culpa depois da realização do comportamento anterior de manter relações sexuais sem preservativo. As atitudes favoráveis ao comportamento, bem como um contexto social mais tolerante, representaram os principais preditores de maior intenção de repetição do comportamento. A variável idade também foi explicativa no modelo obtido. Nesse sentido, os jovens mais velhos, com atitude menos favorável ao uso de preservativo, cujos grupos de convivência (família e amigos) eram mais tolerantes quanto ao não uso, e que apresentavam maior alegria antes e menor medo e culpa após a realização do comportamento, apresentavam maior intenção de voltar a manter relações sexuais sem preservativo no futuro.

O conjunto de variáveis encontrado pode ser indicativo da consolidação de um estilo de vida, reforçado por um contexto de relações (família e amigos) que compartilha as mesmas crenças sobre o uso de preservativo e comportamentos passados com consequências positivas. A prevalência de exposição ao risco de DST-HIV/AIDS encontrada no presente estudo (40\%) pode ser considerada elevada. Embora em 49,4\% dos casos o comportamento tenha ocorrido uma única vez ou poucas vezes, nos $50,6 \%$ dos casos restantes a frequência de realização do comportamento foi em pelo menos metade das vezes que os jovens tiveram relações sexuais. Nesse sentido, é possível afirmar que o comportamento tende a ser habitual. Como as consequências desse comportamento não são, necessariamente, imediatas a sua realização e os temores dos adolescentes são mais associados ao risco de uma gravidez indesejada (Viegas-Pereira, 2000), o fenômeno da habituação precisa ser considerado. No estudo de Teixeira e colaboradores (2006), foi encontrada maior utilização do preservativo na primeira relação sexual, em comparação com a relação sexual mais recente. Tais dados revelam um maior cuidado nas primeiras exposições ao risco, ao mesmo tempo em que indicam menor cuidado quando o não uso de preservativo não ocasionou consequências negativas nas experiências dos jovens, o que decorre do 
passar do tempo (aumento da faixa etária) e da prática relativamente habitual do não uso de preservativo.

Com o aumento da idade, experiência e decorrente avaliação positiva de consequências do não uso de preservativo, as atitudes assumem o papel de maior preditor da intenção de uso de preservativo no futuro. De acordo com Camargo e Botelho (2007), a atitude sobre o uso de preservativo é globalmente favorável entre os adolescentes, todavia, os que já experimentaram relação sexual possuem atitudes menos favoráveis quando comparados aos que não tiveram. Estudo longitudinal realizado por Broaddus, Schmiege e Bryan (2011) também identificaram que ao longo do tempo, com mais experiências sexuais, há uma redução progressiva quanto à intenção de uso de preservativos, independentemente do gênero. Tais aspectos, associados às avaliações otimistas e à não percepção de si como vulnerável para adquirir uma doença (Orlandi \& Toneli, 2008), podem constituir-se em reforçadores para a intenção de repetição.

Estudos brasileiros que relacionaram comportamento sexual de risco entre jovens e os fatores da TCP variam no que tange ao construto atitude. Oliveira, Dias e Silva (2005) e Jemmott III e colaboradores (2007) encontraram correlação significativa entre os componentes atitudinais e a intenção e comportamento de uso de preservativo. Já Matos e colaboradores (2009) e Broaddus e colaboradores (2011) não encontraram influência da atitude em relação à intenção de usar preservativo em adolescentes.

O outro construto da TCP significativo foi a norma subjetiva ou a pressão social. Mesmo resultado foi encontrado em estudos relatados na literatura (Broaddus e colaboradores, 2011; Jemmott III e colaboradores, 2007). Na adolescência, há uma revisão atitudinal em função da inserção em novos grupos e o desejo de consolidar a identidade (Sampaio Filho e colaboradores, 2010). O período é conturbado em ambos os aspectos, atitudes e norma subjetiva. A socialização, especialmente no contexto familiar, faz com que os valores, atitudes e crenças do contexto social sejam assumidos como a forma normativa de comportar-se (Alvarez, 2008).

Quanto aos aspectos emocionais, emoções básicas contribuem de forma importante na explicação da intenção de repetição. Em pesquisa sobre experiência emocional e conduta sexual de risco, Sanchez e colaboradores (2001) identificaram, entre adolescentes madrilenhos, associação de comportamentos de risco com uma ampla gama de experiências emocionais intensas. As emoções avaliadas conjuntamente com as atitudes, dessa forma, influem na seleção de informações que servem de base para a tomada de decisão dos sujeitos em realizar ou não um comportamento de risco, bem como em suas intenções de repeti-lo.

A configuração das variáveis relativas às emoções no modelo obtido está, possivelmente, também atrelada à delimitação da amostra - com experiência de manter relações sexuais desprotegidas no último ano. Há, portanto, uma discrepância entre o conhecimento dos jovens acerca do uso de preservativo como estratégia primordial para prevenção de DST-HIV/AIDS e as emoções que estão atreladas a isso. As atitudes sociais majoritárias no contexto atual são reproduzidas pelos indivíduos, incitando uma resposta racional favorável ao uso. No entanto, a experiência emocional é particular daquele que vivenciou a experiência.

No presente estudo, maior medo e culpa após a realização do comportamento foram as emoções associadas à intenção de realização no modelo obtido. De acordo com estudo de Camargo e Botelho (2007), diante da experiência direta, o jovem compreende a complexidade de manter a racionalidade (optar pelo uso do preservativo) numa situação fortemente emocional.

$\mathrm{O}$ afeto pode ser influenciado pelas experiências anteriores, sendo que quanto mais negativas, menor a probabilidade de realizar novamente o comportamento (Almagia, 2010). Da mesma forma, a antecipação de sentimentos negativos reduz a intenção de realizá-lo novamente (Ferreira, 2011). Já a alegria apresenta uma configuração distinta. Neste estudo, uma maior alegria antes contribuiu para o aumento da intenção de repetição, demonstrando que o estado de ânimo positivo anterior concorre com os sentimentos aversivos posteriores (medo e culpa). Essa emoção positiva, por si só, pode inebriar a razão (Sampaio Filho e colaboradores, 2010). Conjuntamente, as emoções de alegria antes e medo e culpa depois, configuram-se como contraditórias, complexificando o potencial cognitivo de recuperar as emoções associadas ao comportamento como um fator protetivo ante a prática de manter relações sexuais sem preservativo.

A infecção por HIV/AIDS e outras DSTs representa uma ameaça à saúde adolescente, o que pode ser corroborado pelos recentes dados do Ministério da Saúde quanto à incidência de casos de HIV/AIDS notificados entre jovens com idades entre 15 e 24 anos, até metade do ano de 2011, no Brasil (Brasil, 2011). Isso demonstra a atual falta de preocupação com os agravos de ordem sexual, revelando, novamente, que o maior 
temor da juventude ainda está relacionado à gravidez indesejada (Viegas-Pereira, 2000), o que é corroborado por $75 \%$ dos participantes que revelaram utilizar outros métodos que previnem a gravidez, mas não as DST-HIV/AIDS.

Outro resultado que chama a atenção por sua manutenção em diversos estudos, em diferentes faixas etárias da população, através do tempo, é o fato de as relações sexuais desprotegidas serem com parceiros estáveis (Camargo \& Botelho, 2007). A cultura brasileira privilegia a confiança no parceiro como valor, deixando em segundo plano o autocuidado (Matos e colaboradores, 2009; Viegas-Pereira, 2000). Na juventude, esse aspecto adquire proporções ainda maiores, considerando-se os fenômenos inerentes à etapa do ciclo vital, de ilusão de invulnerabilidade (Camargo \& Botelho, 2007) e otimismo ilusório (Viegas-Pereira, 2000). Percebem-se também, em estudo internacional, atitudes menos favoráveis ao uso de preservativos com parceiros estáveis (Empelen \& Kok, 2008).

A medida preventiva, por excelência, é de ordem comportamental e diz respeito, diretamente, ao uso de preservativo. Cabe, portanto, uma intensificação das estratégias de promoção da saúde sexual junto a essa população. Nessa perspectiva, de acordo com os resultados encontrados, os programas precisam investir na consolidação de uma atitude social. Faz-se necessário incorporar formas de sensibilizar emocionalmente os jovens, permitindo uma reflexão sobre os fatores intervenientes nos relacionamentos sexuais e, assim, trazendo ao cenário da vida pessoal o sentido do autocuidado, da autorresponsabilização e um repertório mais ampliado de estratégias para fazer frente aos aspectos imbricados na vida sexual na adolescência.

\section{Considerações finais}

Os resultados confirmam a importância dos aspectos emocionais, juntamente com os cognitivos, sobre a intenção de realização de relações sexuais sem preservativo. Embora os aspectos sociocognitivos tenham sido os mais fortes preditores da intenção de voltar a realizar o comportamento, os aspectos emocionais podem estar sendo negligenciados, dada a dificuldade de seu acesso de forma precisa. Revela-se a importância da contemplação de diversos aspectos da vida dos jovens, indo além de simplesmente o elemento informativo, nos programas de promoção da saúde reprodutiva e prevenção de comportamentos sexuais arriscados entre jovens. Todavia, por ser um estudo transversal, este se limitou a um inquérito retrospectivo, que é afetado pela memória, especialmente relacionada às emoções. Da mesma forma, limita-se ao avaliar aspectos emocionais mediante um instrumento que requer esforços predominantemente cognitivos para seu preenchimento. Torna-se importante considerar, também, a associação do comportamento de não uso de preservativo com outros comportamentos, como é o caso do uso de substâncias, especialmente o álcool, que provoca euforia e diminui o discernimento (Alvarez, 2008), o que não foi avaliado de forma conjunta no presente estudo.

Todos os participantes tinham história pregressa de envolvimento em, pelo menos, uma relação sexual sem uso de preservativo, o que não permitiu análise comparativa com jovens sem essa experiência. A população também é restrita, considerando-se que se trata de jovens inseridos no contexto escolar em um único município da região metropolitana de Porto Alegre.

Sugere-se a ampliação deste estudo para populações distintas, tanto em termos regionais quanto etários, uma vez que há um recrudescimento da epidemia de HIV/AIDS no Brasil (Brasil, 2011) e que os fatores que levam as pessoas a incorrerem em risco são multifacetados. Os aspectos emocionais, nesse sentido, podem ser um indicador a melhor compreensão como um fator de proteção nas intervenções sobre o tema.

\section{Referências}

Ajzen, I. (2002). Perceived behavioral control, self-efficacy, locus of control, and the Theory of Planned Behavior. Journal of Applied Social Psycho$\log y, 32,1-20$.

Ajzen, I., \& Fishbein, M. (Eds.) (1980). Understanding attitudes and predicting social behavior. New Jersey: Prentice Hall.

Almagia, E. B. (2010). Psicologia de la sexualidad. Concepción: Universidad de Concepción. Disponível em: http://pt.scribd.com/doc/35161661/Barra-Almagia-Enrique-Psicologia-de-La-Sexual-Id-Ad. Acesso 22 nov. 2012.

Alvarez, M. L. (2008). Gravidez precoce de estudantes de enfermagem e o consumo de álcool. Revista Latinoamericana de Enfermagem, 16(n'.esp.), 577-583.

Andrade, H. H. S. M., Mello, M. B., Sousa, M. H., Makuch, M. Y., Bertoni, N., \& Faúndes, A. (2009). Changes in sexual behavior following a sex education program in Brazilian public schools. Cadernos de Saúde Pública, 25(5), 1168-1176. 
Armitage, C. J., Conner, M., Loach, J., \& Willets, D. (1999). Different perceptions of control: applying an extended Theory of Planned Behavior to legal and illegal drug use. Basic and Applied Social Psychology, 21(4), 301-316.

Ballester, A. C., \& Asensio, A. B. (2001). La predicción de la conducta a través de los constructos que integran la teoría de acción planeada. Revista Electrónica de Motivación y Emoción, 4(7). Disponível em: http://reme.uji.es/articulos/abreva7191302101/ texto.html. Acesso em 27 out 2012.

Bandura, A. (2000). Exercise of human agency through collective efficacy. Current directions in Psychological Science, 9, 75-78.

Bennet, P., \& Bozionelos, G. (2000). The theory of planned behaviour as predictor of condom use: a narrative review. Psychology, Health \& Medicine, 5(3), 307-326.

Brasil. (2009). Partos em adolescentes caem 30\% em dez anos. Ministério da Saúde. Disponível em: http://portal. saude.gov.br/portal/aplicacoes/noticias/default. cfm? $\mathrm{pg}=\mathrm{dspDetalheNoticia \& id \_ area}=124 \& C O$ _ NOTICIA=10550. Acesso 10 nov. 2012.

Brasil. (2010). AIDS no Brasil. Ministério da Saúde. Disponível em: http://www.aids.gov.br/pagina/ aids-no-brasil. Acesso em 23 de jan.2013.

Brasil. (2011). Boletim epidemiológico de AIDS. Ministério da Saúde. Disponível em: http://www. aids.gov.br/sites/default/files/anexos/publicacao/2011/50652/vers_o_preliminar_69324.pdf. Acesso em 16 nov. 2012.

Broaddus, M. R., Schmiege, S. J., \& Bryan, A. D. (2011). An expanded model of the temporal stability of condom use intentions: gender-specific predictors among high-risk adolescents. Ann. Behav. Med., 42, 99-110.

Caffray, C. M., \& Schneider, S. L. (2000). Why do they do it? Affective motivators in adolescents' decisions to participate in risk behaviours. Cognition and Emotion, 14(4), 543-576.

Camargo, B. V., \& Botelho, L. J. (2007). Aids, sexualidade e atitudes de adolescentes sobre proteção contra o HIV. Revista de Saúde Pública, 41(1), 61-68.

Campo-Arias, A., Ceballo, G. A., \& Herazo, E. (2010). Prevalência do padrão de comportamento de risco para a saúde sexual e reprodutiva em estudantes adolescentes. Revista Latino-Americana de Enfermagem, 18(2), 26-30.

Cruzeiro, A. L. S., Souza, L. D. de M., Silva, R. A., Pinheiro, R. T., Rocha, C. L. A., \& Horta, B. L. (2010). Comportamento sexual de risco: fatores associados ao número de parceiros sexuais e ao uso de preservativo em adolescentes. Ciência \& Saúde Coletiva, 15(Supl. 1), 1149-1158.

Empelen, P., \& Kok, G. (2008). Actio-specific cognitions of planned and preparatory behaviors of condom use among dutch adolescents. Arch. Sex. Behav., 37, 626-640.

Feijó, R. B., \& Oliveira, E. A. (2001). Comportamento de risco na adolescência. Jornal de Pediatria, 77(Supl. 2), S125-S134.

Ferreira, S. I. S. F. V. (2011). Os adolescentes portugueses do 9. ano e o uso do preservativo: atitude, fatores sociais e afectivos (Dissertação de Mestrado). Escola Nacional de Saúde Pública da Universidade Nova de Lisboa, Lisboa, Portugal.

Ferreira, M. M. S. R. S., \& Torgal, M. C. L. F. P. R. (2011). Estilos de vida na adolescência: comportamento sexual dos adolescentes portugueses. Revista da Escola de Enfermagem USP, 45(3), 589-595.

Gouveia, V. V., Pimentel, C. E., Leite, P. R. L., Albuquerque, J. R., \& Costa, T. A. B. (2009). Escala de atitudes frente ao uso de álcool: descrevendo seus parâmetros psicométricos. Psicologia: Ciência e Profissão, 29(4), 672-685.

Jemmott III, J. B., Heeren, G. A., Ngwane, Z., Hewitt, N., Jemmott, L. S., Shell, R., \& O’Leary, A. (2007). Theory of planned behavior predictors of intention to use condoms amog Xhosa adolescents of South Africa. AIDS Care, 19(5), 677-684.

León Rúbio, J. M., \& Medina Anzano, S. (2002). Psicología social de la salud: fundamentos teóricos y metodológicos. Sevilla: Comunicación Social.

Matos, E. B., Veiga, R. T., \& Reis, Z. D. N. (2009). Intenção de uso de preservativo masculino entre jovens estudantes de Belo Horizonte: um alerta aos ginecologistas. Revista Brasileira de Ginecologia e Obstetrícia, 31(11), 574-580.

Mendes, R. D. R., Rodrigues, R. C. M., Cornélio, M. E., Gallani, C. B. J., \& Godin, G. (2010). Desenvolvimento de instrumento para medida de fatores psicossociais determinantes do comportamento de 
atividade física em coronariopatas. Revista da Escola de Enfermagem USP, 44(3), 584-596.

Mérida, J. A. M., \& Jorge, M. L. M. (2010). Análisis comparativo de los principales paradigmas en el estudio de la emoción humana. Revista Electrónica de Motivación y Emoción, 13(34). Disponível em: http://reme. uji.es/articulos/numero34/ article10/resumen. htm. Acesso em 14 nov. 2012.

Neto, F. R. G. X., Dias, M. A. S., Rocha, J., \& Cunha, I. C. K. O. C. (2007). Gravidez na adolescência: motivos e percepções de adolescentes. Revista Brasileira de Enfermagem, 60(3), 279-285.

Oliveira, S. H. S., Dias, M. R., \& Silva, M. I. T. (2005). Adolescentes e AIDS: fatores que influenciam a intenção de uso do preservativo. DST - Jornal Brasileiro de Doenças Sexualmente Transmissiveis, 17(1), 32-38.

Orlandi, R., \& Toneli, M. J. F. (2008). Adolescência e paternidade: sobre os direitos de criar projetos e procriar. Psicologia em Estudo, 13(2), 317-326.

Sampaio Filho, F. J. L., Sousa, P. R. M., Vieira, N. F. C., Nóbrega, M. de F. B., Gubert, F. do A., \& Pinheiro, P. N. da C. (2010). Percepção de risco de adolescentes escolares na relação consumo de álcool e comportamento sexual. Revista Gaúcha de Enfermagem, 31(3), 508-514.

Sanchez, F., Caballero, A., Carrera, P., Blanco, A., \& Pizarro, B. (2001). Sexual risk behaviour and emotional experience. Revue Internacionale de Psychologie Sociale, 14, 7-20.

Serovich, J. M., \& Greene, K. (1997). Predictors of adolescent sexual risk taking behaviors which put them at risk for contracting HIV. Journal of Youth and Adolescence, 26(4), 429-444.

Teixeira, A., Knauth, D., Fachel, J., \& Leal, A. (2006). Adolescentes e uso de preservativos: as escolhas dos jovens de três capitais brasileiras na iniciação e na última relação sexual. Cadernos de Saúde Pública, 22(7), 1385-1396.

Terry, D., Gallois, C. E., \& McCamish, M. (1994). The theory of reasoned action and health care behavior. Em D. J. Terry, C. Gallois e M. McCamish (Eds.), The theory of reasoned action: its application to AIDS-preventive behavior (pp. 1-27). Nova Iorque: Pergamon Press.

Viegas-Pereira, A. P. F. (2000). “AIDS? Tô fora.” Um estudo com adolescentes em duas escolas de Belo Horizonte sobre os fatores que determinam o uso de preservativo na era da AIDS. Disponível em: http://www.abep. nepo.unicamp.br/docs/anais/pdf/2000/Todos/ Posteres/AIDS $\% 20 \mathrm{~T} \% \mathrm{C} 3 \%$ B $4 \% 20$ fora $\% 20-\% 20$ Um\%20Estudo $\% 20 \mathrm{com} \% 20$ Adolescentes....pdf. Acessso em 14 de dez. 2012.

World Health Organization. (2011). Adolescent pregnancy. Disponível em: http://www.who.int/ making_pregnancy_safer/topics/adolescent_ pregnancy/en/index.html. Acesso em 10 jan. 2013.

Recebido em: 05/12/2012

Reformulado em: 18/09/2013 Segunda reformulação em: 03/10/2013

Aprovado em: 10/12/2013 
Sobre as autoras:

Ítala Raymundo Chinazzo é estudante de Psicologia da ULBRA/Canoas e bolsista de Iniciação Científica - CNPq/ PIBIC

Sheila Gonçalves Câmara é psicóloga, doutora em Psicologia (PUCRS), professora do Curso de Psicologia ULBRA/ Canoas e do Programa de Pós-Graduação em Saúde Coletiva da ULBRA e professora do Departamento de Psicologia da UFCSPA.

Deise Gabriela Frantz é estudante de Psicologia da ULBRA/Canoas e bolsista de Iniciação Científica - CNPq.

Contato com as autoras:

Ítala Raymundo Chinazzo

Rua Crispim Machado Silveira, 50

Partenon

Porto Alegre - RS

91530-250

E-mail: italach@gmail.com

Pesquisa financiada pelo CNPq 